This is an Open Access article distributed under the terms of the Creative Commons Attribution-Noncommercial License, which permits unrestricted use, distribution, and reproduction in any noncommercial medium, provided the original work is properly cited.

\title{
MINERALOGY OF CIRCUMSTELLAR AND INTERSTELLAR DUST: THE SPICA POTENTIAL
}

\author{
L.B.F.M. Waters ${ }^{1,2}$ \\ ${ }^{1}$ Astronomical Institute "Anton Pannekoek", University of Amsterdam, Kruislaan 904, 1098 XH Amsterdam, The Netherlands \\ ${ }^{2}$ Instituut voor Sterrenkunde, Katholieke Universiteit Leuven, Celestijnenlaan 200D, B-3001 Leuven, Belgium
}

\section{ABstraCt}

The mid-infared spectral region contains many resonances of dust species that are of astrophysical interest. These resonances can be used to trace the dust cycle in galaxies, starting from the evolved low- and high mass stars, through the interstellar medium to molecular clouds and proto-planetary systems. In particular the mineralogy of circumstellar dust is very rich, reflecting the wide range in physical and chemical conditions that occur near stars in different evolutionary phases. Astromineralogy also offers a quantitative link between the composition of objects in the solar system, and proto-planetary disks and debris disks that are observed around young and mature stars.

Key words: Galaxies: interstellar matter - Stars: formation - Stars: evolved stars - Missions: SPICA

\section{INTRODUCTION}

Solid material or dust is ubiquitous in interstellar space and in circumstellar environments. It plays an important role in the physics and chemistry of interstellar matter, in the stellar winds of evolved stars, and in the formation of stars and planetary systems. Being part of the cycle of matter in galaxies, dust can be used to map this cycle and shed light on the processing of solids in various environments. The chemical composition and lattice structure of solid material can be determined using spectroscopy at mid- and far-infrared wavelengths. This spectral region is very rich in vibrational resonances of abundant dust species, that can be identified using laboratory measurements of cosmic dust analogues. The power of astromineralogy has convincingly been demonstrated by the Infrared Space Observatory (ISO) and more recently by the Spitzer Space Telescope. While ISO was able to reveal for the first time the wealth of infrared resonances in the 5 to $200 \mu \mathrm{m}$ wavelength range, Spitzer with the Infrared Spectrograph IRS revealed the ubiquity of minerals in objects as diverse as brown dwarf disks and the outflows of Active Galactic Nuclei. The recent launch of HERSCHEL has re-opened the 55-200 $\mu \mathrm{m}$ wavelength range for spectroscopy of (cold) solids in space using the PACS instrument, and in the coming years JWST/MIRI will offer unprecedented sensitivity in the $5-28 \mu \mathrm{m}$ wavelength range.
While all these facilities will make or have made groundbraking contributions, it is only with Spica and its spectrographs that the full $5-210 \mu \mathrm{m}$ spectral region will be available again with a unique spatial resolution and sensitivity. This has a huge advantage for astromineralogy studies since many dust species have resonances covering a wide spectral range (and thus temperature), allowing a complete inventory of the dust composition as a function of temperature. Below we discuss the potential of Spica in the area of astromineralogy of cosmic dust.

\section{Astromineralogy OF DUSt}

Table 1 presents an overview of the strongest emission bands detected in the 30-200 $\mu \mathrm{m}$ wavelength range. Here AGB refers to Asymptotic Giant Branch star, P-AGB to post-AGB star (including planetary nebulae), YSO refers to young star, and SFR refers to star forming region. Examples of observed spectra of evolved stars and young stars are given in figures 1 and 3. Also given are the (sometimes uncertain) identifications and the type of object in which the band has been seen. Blending of bands in the 40 and $60 \mu \mathrm{m}$ wavelength range make it difficult to uniquely assign peaks to solids. However, if the entire wavelength range is considered identifications can be more secure. For instance, $\mathrm{MgSiO}_{3}$ (enstatite) has two structures, clino- and ortho-enstatite. These have overlapping bands shortward of $30 \mu \mathrm{m}$ and also in the $40 \mu \mathrm{m}$ region, but can be distinguished at $45 \mu \mathrm{m}$ and in the $65-70 \mu \mathrm{m}$ wavelength range.

A band at $48 \mu \mathrm{m}$ has been seen in the ISO spectra of evolved and young stars. However, the reality of this band is questionable because of uncertainties in the relative spectral response of the ISO-LWS spectrograph.

The $60 \mu \mathrm{m}$ region shows a strong and broad emission band which is likely a blend of crystalline water ice (centered near $60 \mu \mathrm{m}$ ) and a second material with a band near $65 \mu \mathrm{m}$, which may be diopside $\left(\mathrm{CaMgSi}_{2} \mathrm{O}_{6}\right)$, melilite $\left(\mathrm{Ca}_{2} \mathrm{Al}_{2(1-x)} \mathrm{Mg}_{x} \mathrm{Si}_{(1+x)} \mathrm{O}_{7}\right)$ or a combination of these materials (e.g. Chihara et al., 2007; Kemper et al., 2002). The broad $65 \mu \mathrm{m}$ band has been detected in a range of objects, including two star-forming regions (Onaka \& Okada, 2003).

The $91 \mu \mathrm{m}$ band has been attributed to calcite (Kemper et al., 2002) and has so far been detected in two planetary nebulae, in a protostar and in two star-forming re- 

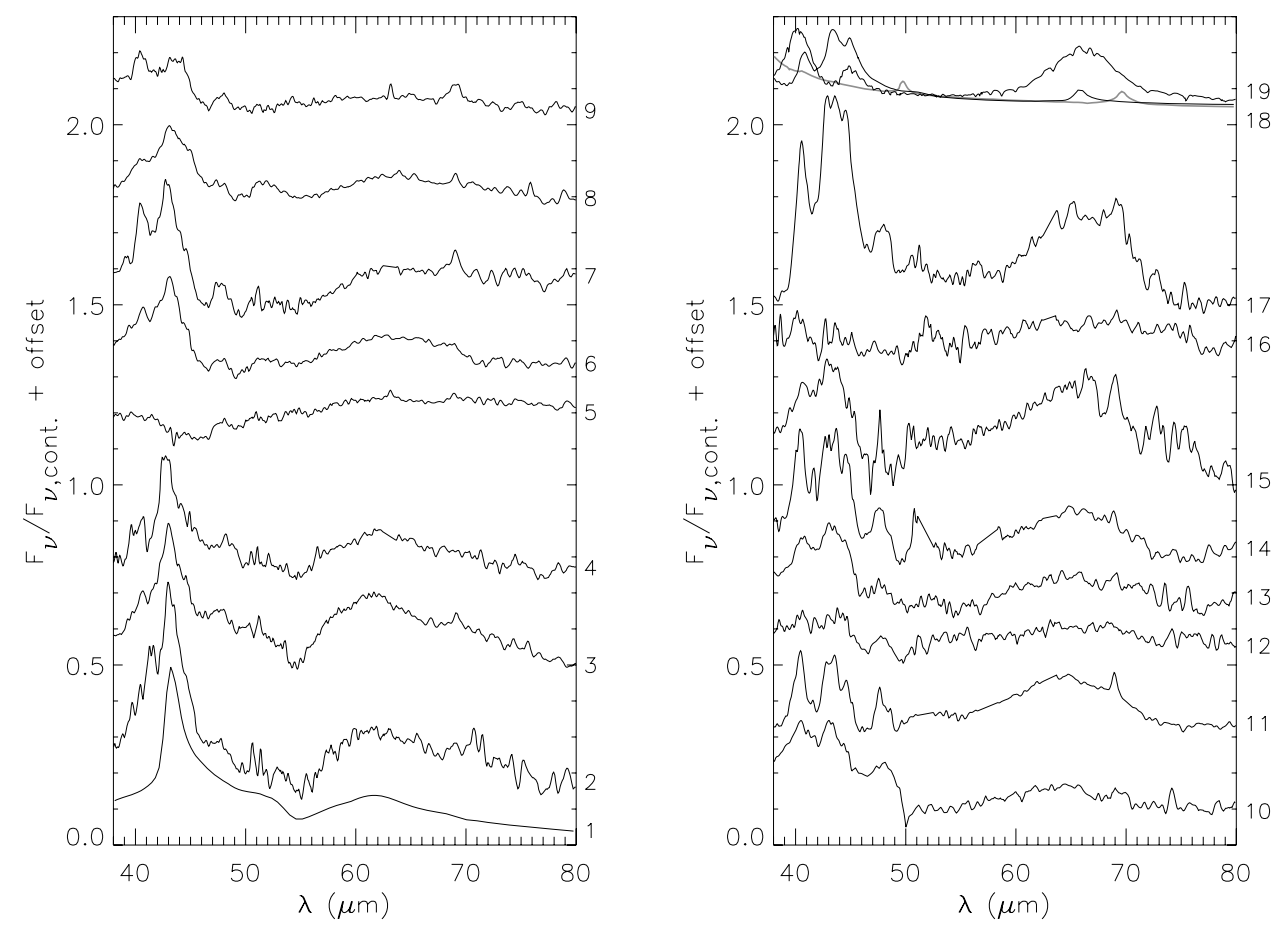

Figure 1. The 38 to $80 \mu \mathrm{m}$ continuum divided spectra for AGB and post-AGB stars with ISO-SWS and LWS spectra of high signal to noise data available over the complete 38 to $80 \mu \mathrm{m}$ spectral range. We selected sources with a reliable $60-65 \mu \mathrm{m}$ band. The number refer to: 1) $50 \mathrm{~K}$ crystalline water ice (Smith et al., 1994), 2) HD161796, 3) HD101584, 4) M 1-92, 5) IRAS 16342-3814, 6) HD179821, 7) CPD 568032, 8) AFGL 5379, 9) HD44179, 10) AFGL 4106, 11) NGC6302, 12) IRAS 17150-3224, 13) OH 26.5+0.6, 14) NGC6537, 15) OH 127+0.0, 16) M 2-9, 17) MWC922, 18) clino-enstatite (Chihara et al., 2002, black line) and forsterite (Koike et al., 2003, grey line), and 19) diopside (Koike et al., 1999). Note that for clearity, offsets have been applied to the individual spectra, and strong forbidden transition lines have been removed from the spectra. Figure taken from Dijkstra, thesis, University of Amsterdam.

gions (Ceccarelli et al., 2002; Onaka \& Okada, 2003). The remarkable ISO spectrum of the young star HD 142527 is shown in figure 3 . This object shows a very broad and strong band centered near 100-110 $\mu \mathrm{m}$ (Malfait et al., 1999), which has been attributed to the hydrous silicate montmorillonite. So far this is the only object in which this band is detected.

It is important to note that the shape and strength of the infrared resonances of many materials is temperature sensitive. A good example of this sensitivity for forsterite is given in figure 2, taken from Koike et al. (2006). The band shifts to shorter wavelength and sharpens with decreasing temperature. This allows for an independent determination of the temperature of this abundant mineral. However, these bands are also very sensitive to chemical composition, and a small inclusion of Fe causes strong band shifts. Observations of several forsterite bands may help to disentangle temperature and chemical composition effects. A second example is the $43 \mu \mathrm{m}$ band of crystalline water ice (Smith et al., 1994).

The dust species discussed so far are all oxygen-rich; Crich AGB stars and P-AGB stars show a very broad band which peaks at $26 \mu \mathrm{m}$ in AGB stars and shifts to about $35 \mu \mathrm{m}$ in planetary nebulae (e.g. Hony et al., 2002). This can be attributed to MgS (Goebel \& Moseley, 1985) at different temperatures.

\section{THE CYCLE OF DUST IN GALAXIES}

The composition of dust in different environments can be used to trace the cycle of matter in galaxies, from the evolved stars that produce dust in large amounts, through the interstellar medium in which dust is destroyed and re-forms, to the inclusion in molecular clouds, protostars and proto-planetary disks. Observations taken with ISO 
Table 1. Overview of dust resonances in the wavelength range 30-200 $\mu \mathrm{m}$ and their identification.

\begin{tabular}{lll}
\hline $\begin{array}{l}\text { wavelength } \\
(\mu \mathrm{m})\end{array}$ & identification & seen in: \\
\hline 30 & $\mathrm{MgS}$ & AGB, P-AGB \\
33.6 & $\mathrm{Mg}_{2} \mathrm{SiO}_{4}$ Forsterite & AGB,P-AGB, YSO \\
$40.5,43$ & $\mathrm{MgSiO}_{3}$ c-Enst, o-Enst & AGB, P-AGB \\
45 & $\mathrm{MgSiO}_{3}$ c-Enst & AGB, P-AGB \\
$48 ?$ & $?$ & AGB, PAGB, YSO \\
43,60 & cryst. $\mathrm{H}_{2} \mathrm{O}$ ice & AGB, p-AGB, YSO \\
65 (broad) & $\mathrm{CaMgSi}_{2} \mathrm{O}_{6}$ Diopside? & P-AGB, SFR \\
65 (narrow) & $\mathrm{MgSiO}_{3}$ c-Enst? & MWC922 \\
69 & $\mathrm{Mg}_{2} \mathrm{SiO}_{4}$ Forsterite & AGB,P-AGB \\
91 & $\mathrm{CaCO}_{3}$ Calcite & P-AGB, YSO, SFR \\
$100-110$ & $\mathrm{Hydrous} \mathrm{silicate?}$ & HD142527 \\
\hline
\end{tabular}

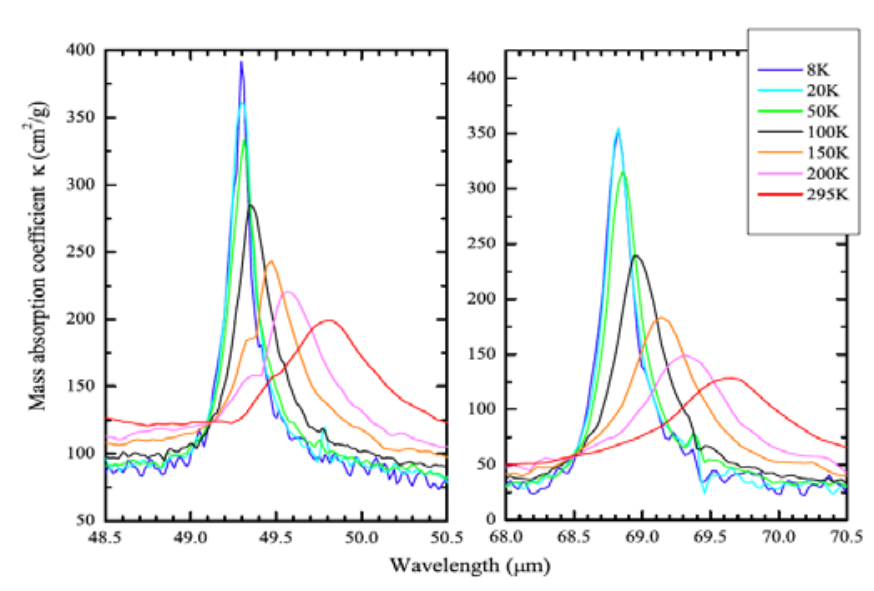

Figure 2. Laboratory measurements of the 49 and $69 \mu \mathrm{m}$ forsterite bands as a function of temperature, taken from Koike et al. (2006) .

and Spitzer show a very rich circumstellar mineralolgy in evolved stars (table 1) while interstellar dust in our galaxy shows only a few dust species (amorphous silicate, amorphous carbon, SiC). The disks surrounding young stars show an increased diversity of dust species with respect to the ISM, pointing to substantial dust processing in these disks.

\subsection{DUST IN EVOLVED STARS}

The composition of dust detected in evolved stars shows a wide range of compositions, that reflect the physical and chemical conditions in the dust forming layers. Oxygenrich Asymptotic Giant Branch (AGB) stars and Red Supergiants (RSG) with low mass loss rates show evidence for simple oxides and amorphous silicates (e.g. Posch et al., 2002; Verhoelst et al., 2009). Stars with high mass loss rates show amorphous and crystalline silicates as well as crystalline water ice. The formation of crystalline silicates may be related to high densities in the dust forming regions . Similarly, gas-phase water condenses in the outflow only at high densities. C-rich AGB stars show amorphous silicates, $\mathrm{SiC}$, and $\mathrm{MgS}$.

The spectra of oxygen-rich AGB and P-AGB stars shown in figure 1 have been ordered in a sequence of increasing wavelength of the broad " $60-65 \mu \mathrm{m}$ " band. The band shifts from about 60 to $65 \mu \mathrm{m}$. The sources whose band peaks near $60 \mu \mathrm{m}$ are dominated by crystalline water ice (also seen at $43 \mu \mathrm{m}$ ), while sources peaking near $65 \mu \mathrm{m}$ show prominent crystalline silicate bands. An interesting trend can be noted between the shape of the IR spectra and the geometry of the dust shell. Stars with strong water ice bands are generally stellar wind sources, while sources with strong crystalline silicate emission tend to have disk-like geometries (Molster et al., 1999). However there are exceptions to this trend, e.g. HD101584. Many of the evolved stars with a disk-like geometry are member of a binary system (e.g. van Winckel, 2003), in which a disk of gas and dust has formed around the binary system. The dust processing in these disks (aggregation, settling and crystallization) is similar to that seen in the proto-planetary disks surrounding young stars.

\subsection{THE INTERSTELLAR MEDIUM}

The dust produced by evolved stars eventually mixes with that of the interstellar medium. A rich mineralogy of interstellar dust may thus be expected, but in fact only few species have been found in the ISM from infrared spectroscopy, i.e. amorphous silicates, amorphous carbon (or graphite) and $\mathrm{SiC}$, the latter only in the direction of the galactic centre (Min et al., 2007). (The list is longer when meteoritic pre-solar grains are included). In only a few cases dust bands of other species have been detected in the ISM (Onaka \& Okada, 2003). A comparison between the typical interstellar dust destruction timescale and the observed abundance of interstellar dust shows that dust must be efficiently re-formed in the ISM (Jones et al., 1994), but where this occurs (diffuse ISM, molecular clouds, protostellar cores) is not known.

In starburst galaxies with a high dust injection rate, "stardust" may be abundant in their interstellar medium. Indeed, Spoon et al. (2006) detect crystalline silicates in such galaxies, possibly due to mass loss by RSG and massive AGB stars.

\subsection{DUST IN YOUNG STARS}

Cold molecular clouds show infrared extinction curves that are markedly different from that of the diffuse ISM, especially in the ratio $\mathrm{E}(\mathrm{J}-\mathrm{K}) / \tau_{9.7}$, where $\tau_{9.7}$ is the optical depth of the $9.7 \mu \mathrm{m}$ silicate band (Chiar et al., 2007). This indicates that dust processing is ongoing in these dense environments. However, the shape of the $9.7 \mu \mathrm{m}$ silicate absorption band does not change strongly, so that 


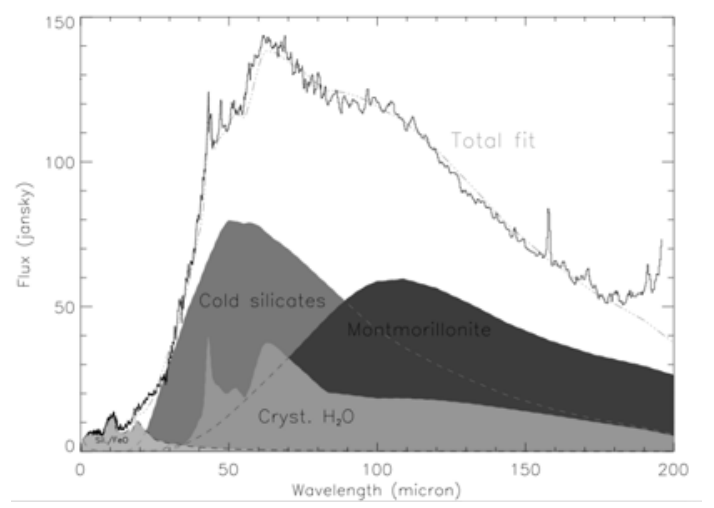

Figure 3. ISO spectrum of the young herbig Ae/Be star HD142527 showing a rich mineralogy of the cold dust. Identifications of resonances are indicated (Malfait et al., 1999).

the cause of the change in the extinction law remains unclear: growth or changes in the chemical composition of the dust.

More spectacular changes in the dust composition occur in the proto-planetary disks that surround low- and intermediate mass stars. These disks can show very high abundances of forsterite and enstatite, as well as crystalline water ice. Observations at high spatial resolution show that crystalline silicate abundance is high in the innermost disk regions, where temperatures are high enough for amorphous silicates to anneal (Leinert et al., 2004). However, many disks also show the presence of cold crystalline silicates, that must imply either efficient radial mixing or the local production of crystalline silicates e.g. by shocks. Depending on the thermal history of the dust (gasphase condensation or thermal annealing) different abundances of forsterite and enstatite are expected. The relative abundance of clino- and ortho-enstatite may also be used as a tracer of the formation history of these minerals. Slow condensation favours ortho-enstatite while rapid cooling favours clino-enstatite (Koike et al., 2006). The spatial distribution of crystalline silicates can thus be used to infer information about global processes that occur in the disk.

A remarkable mineralogy has been found in the ISO spectrum of the intermediate mass pre-main-sequence star HD142527, see figure 3. The ISO spectrum shows evidence for crystalline silicates, crystalline $\mathrm{H}_{2} \mathrm{O}$ ice, and possibly hydrosilicates (Malfait et al., 1999). Observations with the VLT Interferometer show that the inner disk silicates are 100 per cent crystalline (van Boekel et al., 2004). The disk of HD142527 shows a large gap extending to $130 \mathrm{AU}$ (Verhoeff et al., in preparation; see also Fujiwara et al. (2006)), which may be the result of planet formation. The remarkable mineralogy of the cold dust in the outer disk may be related to the formation of a gas giant planet, causing the production of small grains through collisions between larger bodies. The mineralogy of the cold dust may thus reflect that of the parent bodies and the effects of impacts on the composition of the material. In the case of HD 142527 the location of the dust suggests the parent bodies may have been similar to solar system Kuiper belt objects.

\section{ACKNOWLEDGEMENTS}

We thank Dr. Koike for permission to use figure 2, and A. Verhoeff, M. Min, H. Fujiwara and E. Pantin for discussions on the nature of the disk in HD 142527.

\section{REFERENCES}

Ceccarelli C., Caux E., Tielens A.G.G.M., et al.: 2002, $A \& A$ 395, L29

Chiar J.E., Ennico K., Pendleton Y.J., et al.: 2007, ApJ 666, L73

Chihara H., Koike C., Tsuchiyama A.: 2007, A\&A 464, 229

Chihara H., Koike C., Tsuchiyama A., Tachibana S., Sakamoto D.: 2002, A $\& A$ 391, 267

Fujiwara H., Honda M., Kataza H., et al.: 2006, ApJ 644, L133

Goebel J.H., Moseley S.H.: 1985, ApJ 290, L35

Hony S., Waters L.B.F.M., Tielens A.G.G.M.: 2002, $A \& A$ 390,533

Jones A.P., Tielens A.G.G.M., Hollenbach D.J., McKee C.F.: 1994, ApJ 433, 797

Kemper F., Molster F.J., Jäger C., Waters L.B.F.M.: 2002, A\&A 394, 679

Koike C., Chihara H., Tsuchiyama A., et al.: 2003, $A \& A$ 399, 1101

Koike C., Mutschke H., Suto H., et al.: 2006, A\&A 449, 583

Koike C., Tsuchiyama A., Suto H.: 1999, Proc. of the 32nd ISAS Lunar and Planetary Symposium 32, 175

Leinert C., van Boekel R., Waters L.B.F.M., et al.: 2004, A\&A 423, 537

Malfait K., Waelkens C., Bouwman J., de Koter A., Waters L.B.F.M.: 1999, A $\& A$ 345, 181

Min M., Waters L.B.F.M., de Koter A., et al.: 2007, $A \& A$ 462,667

Molster F.J., Yamamura I., Waters L.B.F.M., et al.: 1999, Nature 401, 563

Onaka T., Okada Y.: 2003, ApJ 585, 872

Posch T., Kerschbaum F., Mutschke H., Dorschner J., Jäger C.: 2002, A\& A 393, L7

Smith R.G., Robinson G., Hyland A.R., Carpenter G.L.: 1994, MNRAS 271, 481

Spoon H.W.W., Tielens A.G.G.M., Armus L., et al.: 2006, ApJ 638, 759

van Boekel R., Min M., Leinert C., et al.: 2004, Nature 432,479

van Winckel H.: 2003, ARA\&A 41, 391

Verhoelst T., van der Zypen N., Hony S., et al.: 2009, $A \& A$ 498, 127 